This item was submitted to Loughborough's Research Repository by the author.

Items in Figshare are protected by copyright, with all rights reserved, unless otherwise indicated.

\title{
Modeling and analysis of LTE connectivity in a high mobility vehicular environment
}

PLEASE CITE THE PUBLISHED VERSION

https://doi.org/10.1016/j.compeleceng.2018.04.007

\section{PUBLISHER}

(c) Elsevier

\section{VERSION}

AM (Accepted Manuscript)

\section{PUBLISHER STATEMENT}

This work is made available according to the conditions of the Creative Commons Attribution-NonCommercialNoDerivatives 4.0 International (CC BY-NC-ND 4.0) licence. Full details of this licence are available at: https://creativecommons.org/licenses/by-nc-nd/4.0/

\section{LICENCE}

CC BY-NC-ND 4.0

\section{REPOSITORY RECORD}

Burbano-Abril, Andres, R.M. Edwards, Victor Rangel, Raul Aquino-Santos, Miguel Lopez-Guerrero, Roy S. Kalawsky, and Mohammadreza Behjati. 2019. "Modeling and Analysis of LTE Connectivity in a High Mobility Vehicular Environment”. figshare. https://hdl.handle.net/2134/32773. 


\title{
Modeling and analysis of LTE connectivity in a high mobility vehicular environment
}

\author{
A. Burbano-Abril ${ }^{\mathrm{a}, *}$, R. M. Edwards ${ }^{\mathrm{b}}$, V. Rangel-Licea ${ }^{\mathrm{a}}$, R. Aquino-Santos ${ }^{\mathrm{c}}$, M. Lopez-Guerrero ${ }^{\mathrm{d}}$, R.S. \\ Kalawsky $^{\mathrm{b}}$, M. Behjati ${ }^{\mathrm{b}}$ \\ ${ }^{a}$ School of Engineering, National Autonomous University of Mexico, Valdés Vallejo Bldg., 04510, Mexico City, Mexico. \\ ${ }^{b} 5 G$ Research Centre, Loughborough University, Loughborough, UK. \\ ${ }^{c}$ School of Telematics, University of Colima, Colima, Mexico. \\ ${ }^{d}$ Department of Electrical Engineering, Metropolitan Autonomous University, Mexico City, Mexico.
}

\begin{abstract}
Long-Term Evolution (LTE) technology has several features that make it an attractive alternative to be used in vehicle-to-infrastructure communications for intelligent transportation systems. However, before LTE can be widely used in this context, a number of analysis must provide convincing evidence that critical network functions (e.g. resource allocation strategies) yield adequate performance. To this end, in this work, we introduce a Markov-chain based model for LTE downlink channel quality, a prime factor affecting performance. Our model comes from the analysis of a large number of measurements of LTE Cell-Specific Reference Signals that were collected through a crowdsourcing application on a motorway in the UK. The model is intended to be used in performance evaluation studies and we exemplify its use with a case study, where we estimate the downlink transmission capacity of an LTE network. We also discuss other potential applications.
\end{abstract}

Keywords:

Benchmarking, Channel Quality Indicator, Finite-State Markov Chain, LTE, Vehicular Environment.

\section{Introduction}

Analysis of Long-Term Evolution (LTE) for vehicle-to-infrastructure (V2I) communications has become an important research area in recent years. This interest arises from the advantages of LTE in comparison with $802.11 \mathrm{p}$ in terms of ubiquitous deployment, large cell-coverage and continuous development by the 3rd Generation Partnership Project (3GPP) [1, 2].

An important performance-related parameter in LTE is the quality of the downlink channel. In LTE, downlink-channel quality estimations are performed through measurements of a type of control signals called

\footnotetext{
* Corresponding author

Email addresses: bburbano@comunidad.unam.mx (A. Burbano-Abril), r.m.edwards@lboro.ac.uk (R. M. Edwards), victor@fi-b.unam.mx (V. Rangel-Licea), aquinor@ucol.mx (R. Aquino-Santos), milo@xanum.uam.mx (M. Lopez-Guerrero), R.S.Kalawsky@lboro.ac.uk (R.S. Kalawsky), M.Behjati@lboro.ac.uk (M. Behjati) 
Cell-Specific Reference Signals (CRS) [3]. To estimate the channel quality, an LTE User Equipment (UE) measures the Signal to Noise Ratio of CRS (CRS-SNR) and converts such reading to a value of Channel Quality Indicator (CQI). This CQI value is reported to the base station of the LTE operator (known as eNodeB), where it is used by a scheduling algorithm to perform resource allocation and link adaptation procedures.

Given the relevance of these procedures, a methodology for evaluating the effect of CQI values on the performance of scheduling algorithms for V2I communications is required. To this end, a model that characterizes the CQI values reported by mobile UEs in vehicular environments has yet to be developed. For this reason, the main objective of this paper is to introduce a downlink-channel quality model, which could be used to evaluate the performance of resource allocation algorithms in LTE for V2I communications.

Creation of such a model requires a prior step consisting of collecting a large number of measurements of LTE downlink performance, to be recorded by mobile UEs in vehicular environments. One method to collect such data is to make use of application-specific systems (i.e., hardware and software) in extensive measurement campaigns. This is the approach that was used in available studies [4-10]. In an alternative way, commonly used smartphones can also be used to collect measurements in a distributed and cooperative manner. This is the basis of the crowdsourcing-based approach for data collecting and it is becoming a feasible alternative due to the wide availability of smartphones with adequate computing power, low cost and reasonable measurement accuracy [11]. However, extracting valid measurements from crowdsourced data in vehicular environments, and specially under high mobility conditions, is not a straightforward task. For this reason, the first contribution of this paper is to propose an analysis methodology of CRS measurements collected through a crowdsourcing application to evaluate the performance of LTE for V2I communications.

Not only do available studies make use of specialized systems for data gathering, but they also focus on analyzing measurements of downlink channel quality without proposing a model. In order to address this limitation, the second contribution of this paper is to provide a novel method to model channel quality for UEs in a vehicular environment by means of a finite-state Markov chain (FSMC). The FSMC is used to model the CQI levels obtained from CRS-SNR measurements collected through a crowdsourcing application, which, to the best of our knowledge, has not been explored before.

The rest of the paper is organized as follows. Section 2 discusses related literature. In Section 3, the measurement methodology is described. In Section 4, the method to model CQI by means of an FSMC is presented and the results are discussed in Section 5. In Section 6 a model to estimate the downlink transmission capacity is derived. In Section 7 this model is combined with the results from the FSMC to estimate the downlink transmission capacity in the analyzed scenario. Finally, Section 8 discusses potential applications and limitations of the FSMC and Section 9 presents the conclusions. 


\section{Related work}

Due to the current popularity of smartphones, several works have applied the crowdsourcing-based approach for data collection. These data are used, in turn, for the analysis and modeling of different performance indicators of cellular communication systems. None of these models, however, is intended to represent downlink channel quality.

In [12], a crowdsourcing application was developed and made available to android users for collecting throughput and packet flow measurements of an LTE network. In this study, collected measurements exhibited high variability in throughput of cellular networks as a consequence of buffering and queuing processes. These results show that crowdsourced measurements can be used as feedback when configuring network protocols or implementing operator policies.

Other works have made use of crowdsourced measurements to explore how diverse factors affect throughput in cellular networks. For instance, authors in [13] studied how received signal strength, geographical location of users and time of day are related to throughput. This piece of work also shows that evaluating network performance, in terms of throughput, requires a comprehensive analysis in order to provide results that are accurate enough and thus, can be used to assess and improve network performance.

The work reported in [14] also analyzed crowdsourced measurements to explore the relationships between the throughput achieved by end users and factors such as time of day, signal strength and signal to noise ratio. In this work, measurements are used to create a linear regression model for predicting downlink throughput. Similar to [13], obtained results show that predicting throughput is not a straightforward task. However, it shows that, in comparison with the rest of collected parameters, the signal to noise ratio measured by UEs is far more correlated to downlink throughput.

When analyzing the performance of the LTE downlink for V2I communications, several pieces of research have proposed the use of measurements collected either through specialized hardware/software or applications installed in smartphones.

In [4], the downlink-channel performance is analyzed through measurements of signal strength, signal to noise ratio and throughput collected by UEs moving at a speed of $80 \mathrm{~km} / \mathrm{h}$, under low traffic conditions and considering a network optimized for benchmarking. During the analysis, good channel conditions were observed, resulting on an average downlink throughput of $33 \mathrm{Mb} / \mathrm{s}$ when Multiple Input Multiple Output (MIMO) $2 \times 2$ was implemented. This analysis demonstrates that, for UEs in vehicular environments, signal to noise ratio remains as the most influencing factor in both uplink and downlink throughput of LTE users.

A similar analysis can be found in [5], where a comparison in terms of channel quality and throughput is performed between UEs moving at a speed of $70 \mathrm{~km} / \mathrm{h}$ and static UEs. In this study, measurements are collected by a single user under unspecified load conditions, which compromise the reliability of the measured downlink performance. However, the results do provide a valuable comparison between the performance of 
mobile and static UEs, which as expected, shows that UEs in the static setup experienced considerably better channel conditions leading to up to $70 \%$ more throughput $(17 \mathrm{Mb} / \mathrm{s})$ when compared with mobile UEs $(10 \mathrm{Mb} / \mathrm{s})$.

A more recent study can be found in [6], where an LTE-Advanced (LTE-A) network was evaluated under different load conditions and at a lower speed $(50 \mathrm{~km} / \mathrm{h})$ than the ones considered in [4] and [5]. In the study, channel quality and throughput were assessed while carrier aggregation (an LTE-A feature) was implemented with two carriers of $10 \mathrm{MHz}$ and $15 \mathrm{MHz}$. During the test, each carrier achieved an average throughput of $17 \mathrm{Mb} / \mathrm{s}$ and $30 \mathrm{Mb} / \mathrm{s}$, respectively. In a similar way to other studies, this work shows how channel quality and downlink performance are degraded under mobility conditions.

Other studies have focused on different performance indicators of LTE for V2I communications based on the analysis of collected measurements. This is the case of [7], where coverage and handover are evaluated, and [8], where delay is analyzed for multimedia applications. Moreover, other works have proposed the use of measurements for modeling certain characteristics of the communication channel. For instance, in [9] the performance of MIMO antennas is evaluated whereas in [10] a model for assessing the impact of shadowing in vehicular environments was obtained.

\section{Measurement methodology}

This section describes various aspects related to the data collection as well as other additional considerations that were taken into account in order to make the collected measurements suitable for analysis.

\subsection{Description of the vehicular environment and collection method}

The dataset used in this research was collected during six months (from June to November 2015) through the crowdsourcing platform OpenSignal. OpenSignal provides an app as well as a website for collecting and retrieving network performance statistics, respectively. The app records the performance statistics of connections between mobile UEs and the eNodeB. The website, in turn, provides aggregated statistics presented in the form of coverage maps.

In the collected dataset there were measurements from all available operators in the surveyed area. However, for this study, from the original dataset we extracted the subset corresponding to the operator with the largest number of CRS measurements, thus producing a new dataset with approximately 300,000 measurements.

From the selected subset, samples were filtered as described below based on the location reported by UEs at the moment of the measurement. The location was obtained by the UEs either by using the built-in GPS or by triangulation of known eNodeBs. From the collected samples, only users with a reported location constrained to be on the M1 motorway were considered. The M1 motorway is a south-north route with a 
length of 193.6 miles (311.6 kilometers) that connects London to Leeds. Figure 1 shows the map of the M1 motorway.

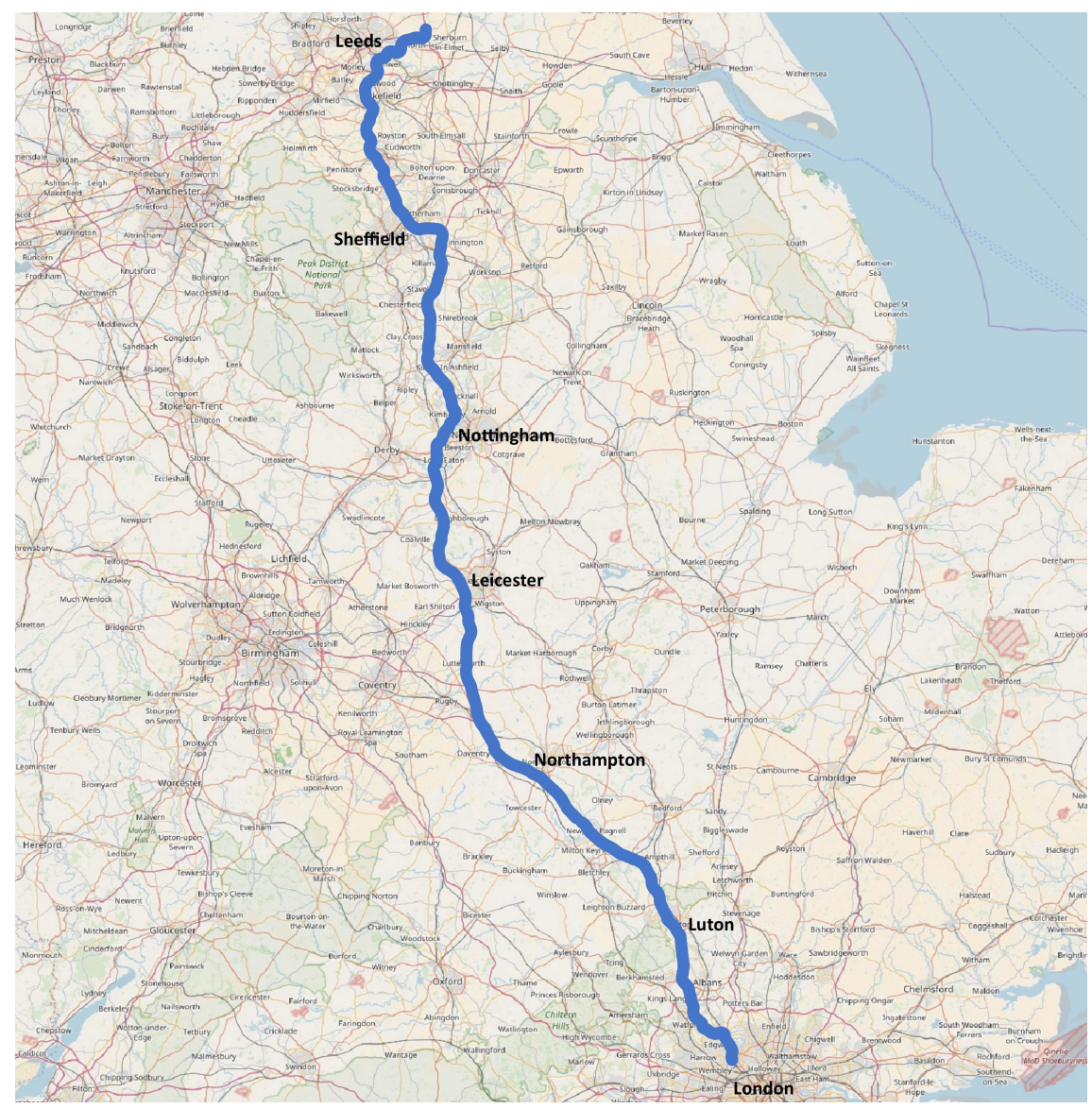

Figure 1: Map of M1 motorway [15].

In Figure 2, a $1.3 \mathrm{~km}$ section of the motorway with approximately 1650 samples is shown. The location precision of the samples is illustrated by circles, with smaller circles representing a more accurate location estimation.
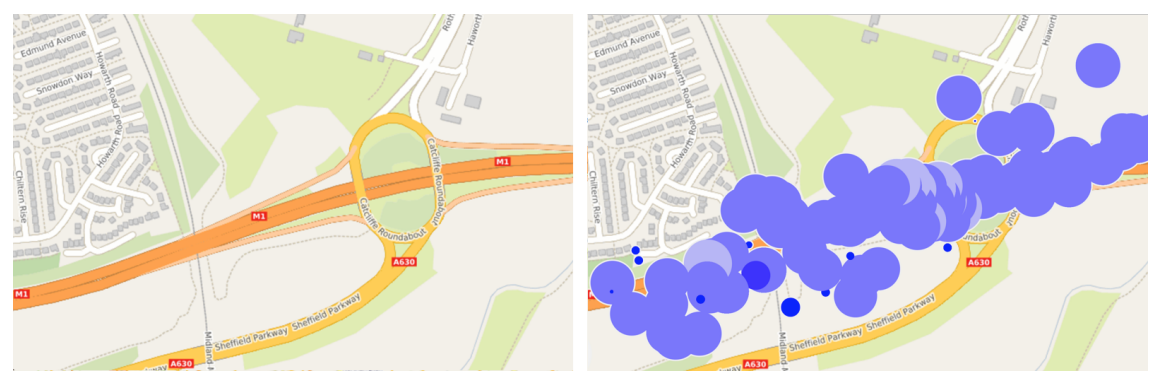

Figure 2: M1 section of approximately $1.3 \mathrm{~km}$ long. Road layout (left). Approximately 1650 measurements (right). [15]. 
In Figure 2, a few houses can be observed at the north-west of the section. However, scenarios like this were not usual in the analyzed motorway. This is due to the fact that planning laws for motorways in the UK prevent buildings from being built close to major roads as a measure to avoid the negative effects of noise and pollution on people's health.

Regarding the location-related characteristics of the measurements, two comments are worth mentioning. First, the great majority of measurements were most likely collected by UEs in vehicles, since pedestrians have very limited access to motorways. And second, the measurements were collected from vehicles that were either moving at average speeds for motorways or temporarily stopped in traffic jams.

\subsection{Collected data}

As previously mentioned, the dataset corresponding to the selected operator originally consisted of approximately 300,000 records of CRS measurements. These data were processed as explained below.

Each one of the records in the dataset contained several parameters related to the measured CRS. Among these parameters, the CRS-SNR was selected to be studied considering that it is the main parameter involved in channel quality estimation. The observed values for CRS-SNR were found to lie within the interval $-20 \mathrm{~dB}$ to $30 \mathrm{~dB}$. Although the range for this parameter is not defined in the standard, measurements performed in other studies such as in [5] and [6] agree with these values.

The time and location of the measurements were also specified in the records. They contained timestamps rounded to the nearest minute and location in terms of latitude and longitude. In order to show the temporal characteristics of the measurements, Figure 3 (left) presents the time distribution of the collected samples, where it can be seen that the majority of measurements were taken during working hours, with peaks in the morning (7 to 9 a.m.) and in the evening (4 to 6 p.m.) during the rush hours.

As for location, Figure 3 (right) provides the number of measurements collected in each $\mathrm{km}$ along the motorway. From this figure, it can be observed that apart from London, which is the major city in the UK, there is a roughly similar number of measurements per $\mathrm{km}$.

\subsection{Filtering method}

The original dataset was provided and pre-filtered by OpenSignal according to the geographic location of the measurements. Therefore, only samples with coordinates (latitude and longitude) constrained to be within a range of 200 meters from each side of the motorway were considered. This range corresponds to the smallest resolution (in terms of location) that can be provided by the OpenSignal platform. 

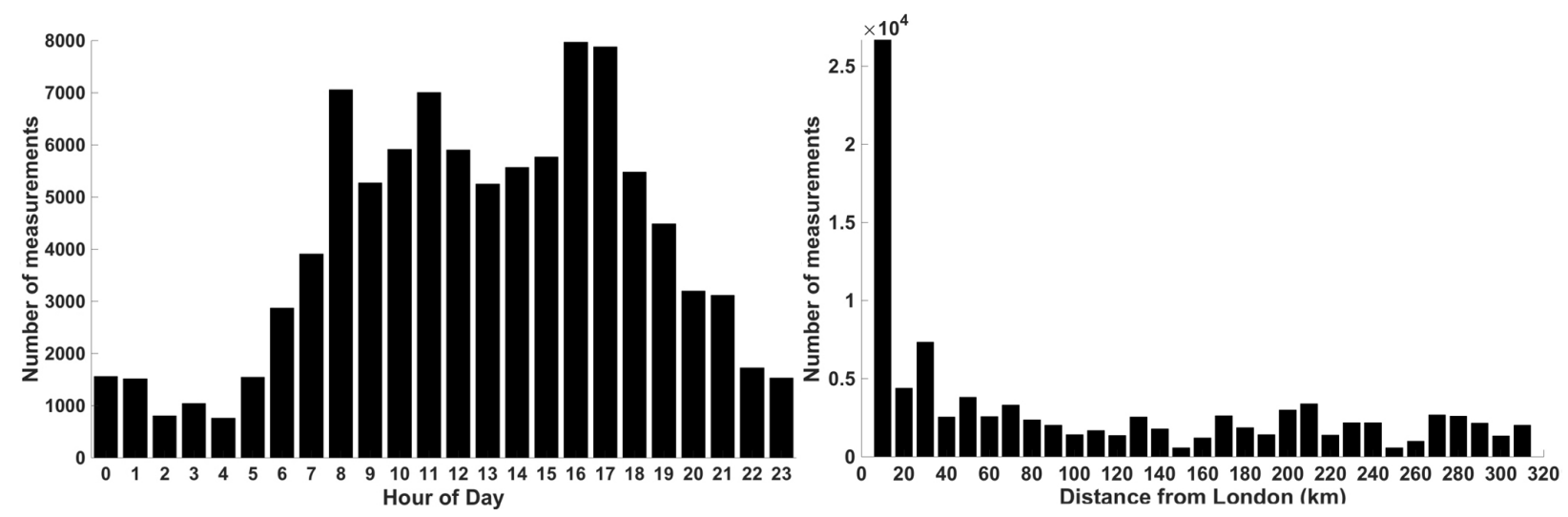

Figure 3: Left, distribution of measurements per hour of day; right, spatial distribution of measurements.

From this dataset, the records from the selected mobile operator were filtered to avoid inconsistencies and improve the reliability of the results. In order to carry out this filtering process, location, type of connection of the UE, and CRS-SNR values were validated as described below.

First, only measurements from mobile users were considered. For this reason, the samples located within a range of 200 meters near the 12 service stations of the M1 motorway [16] were excluded. It was considered that these samples came from users that were either on a fixed location or moving at relatively slow speeds.

In addition, the type of connection (Wi-Fi or mobile) reported by the UEs was also analyzed. All samples from users reporting a Wi-Fi connection at the time of the measurement were not considered. The reason is that it is likely that these users were located at a fixed location and therefore, the CRS-SNR was not measured from a moving vehicle.

Finally, CRS-SNR values falling out of the normal operating range were discarded. For this purpose, the results in [5] and [6] were used as a reference since the standard does not define this range. Outliers in CRS-SNR values were likely logged during handovers between LTE and other radio access technologies.

Filtering was applied to all measurements collected during the time span mentioned above. It is important to mention that subsets of data corresponding to different periods of time were also analyzed and no significant differences among the statistical properties of the compared subsets were found.

After applying the filtering method just described, 92,525 records of measurements scattered over 16,000 different locations on the motorway were obtained. The following section describes how this dataset was used to model the signal quality measured by mobile UEs along the analyzed motorway.

\section{CQI modeling using a finite-state Markov chain}

This section describes the methodology for modeling the CQI using a FSMC. For the sake of clarity, this method is discussed in three parts. First, a method for resampling the original CRS-SNR measurements, 
in order to obtain regularly spaced sampling points, is explained. Then, the mapping between resampled CRS-SNR measurements and CQI levels is described. Finally, the process to obtain the FSMC is presented.

\subsection{Resampling method}

In order to model the CQI trace by means of an FSMC, the original CRS-SNR measurements were resampled at regularly spaced points along the road. This procedure was necessary since we had no control of the crowdsourcing application and therefore, no control over the time or position at which a user created a record. Here we describe a way to cope with this problem.

In the first step, the motorway was divided into $r$ regularly spaced zones of the same area. The area of these zones was chosen as to contain a number of samples large enough to allow the extraction of its statistical properties and also considering that all points within each zone should share similar statistical properties. For this reason, the diameter of each zone in all cases was set to $1 \mathrm{~km}$, yielding a total of 310 zones.

From the 310 zones that were created, 241 of them had 50 or more samples. In these cases, we theorized that the distribution of observed values within zone $n$ (where $n=1, \ldots, r$ ) could be represented by using a Gaussian distribution whose mean value $\mu_{n}$ and variance $\sigma_{n}^{2}$ could be estimated from the observations corresponding to that zone. We used this statement as the null hypothesis to be tested by the KolmogorovSmirnov test [17] to determine the goodness of fit of our assumption in each zone. The results showed that 225 out of the 241 zones passed the test, at the 0.05 significance level, meaning that they contained samples with CRS-SNR values that can be considered to come from a Gaussian distribution. Based on these results, each one of these zones, with more than 50 samples, was modeled as a Gaussian distribution. This process allowed us to preserve the statistical properties of each section of the motorway.

In the case of zones with less than 50 samples their mean and variance were not estimated from the sample set. In such cases both statistical descriptors were interpolated from adjacent zones as follows.

Let $X_{1}, Z$ and $X_{2}$ be three random variables representing the statistics of three contiguous zones, being $Z$ the one in the middle whose PDF is unknown. Let us also consider that $X_{1}$ and $X_{2}$ are Gaussian whose parameters are known, that is $X_{1} \sim N\left(m_{1}, \sigma_{1}^{2}\right)$ and $X_{2} \sim N\left(m_{2}, \sigma_{2}^{2}\right)$. It is proposed to consider that the statistical properties of $Z$ can be estimated as the average of $X_{1}$ and $X_{2}$, i.e.

$$
Z=\frac{X_{1}+X_{2}}{2}
$$

It can be easily shown, from the theory of jointly Gaussian random variables [18], that $Z$ is also Gaussian (i.e., $\left.Z \sim N\left(m_{Z}, \sigma_{Z}^{2}\right)\right)$ with mean

$$
m_{Z}=\frac{1}{2} m_{1}+\frac{1}{2} m_{2}
$$


and variance

$$
\sigma_{Z}^{2}=\frac{1}{4} \sigma_{1}^{2}+\frac{1}{2} \operatorname{COV}\left(X_{1}, X_{2}\right)+\frac{1}{4} \sigma_{2}^{2} .
$$

In this way, the parameters of $Z$ can be estimated from the ones corresponding to adjacent zones.

This procedure was applied to zones with less than 50 samples surrounded by zones with more than 50 samples. In total 38 zones were interpolated in this way, which in addition to the 241 zones mentioned before, accounted for $90 \%$ of the total length of the motorway.

In cases where two or more consecutive zones with less than 50 samples were found (i.e., 31 zones), it was assumed that LTE coverage was unavailable. In the analysis, these zones were assigned the SNR for CQI 0, which is used by UEs to report the out-of-coverage state.

Having modeled each zone, the resampling was performed considering a total of $m$ regularly spaced sampling points $S P_{i}$ for $i=1, \ldots, m$ for the entire motorway. The resampling distance was set to 20 meters, yielding a total of $50 \mathrm{CRS-SNR}$ samples per zone and, therefore, $15500 \mathrm{CRS}-\mathrm{SNR}$ samples in total. Each one of these points represents a single CRS-SNR measurement, as illustrated in Figure 4.

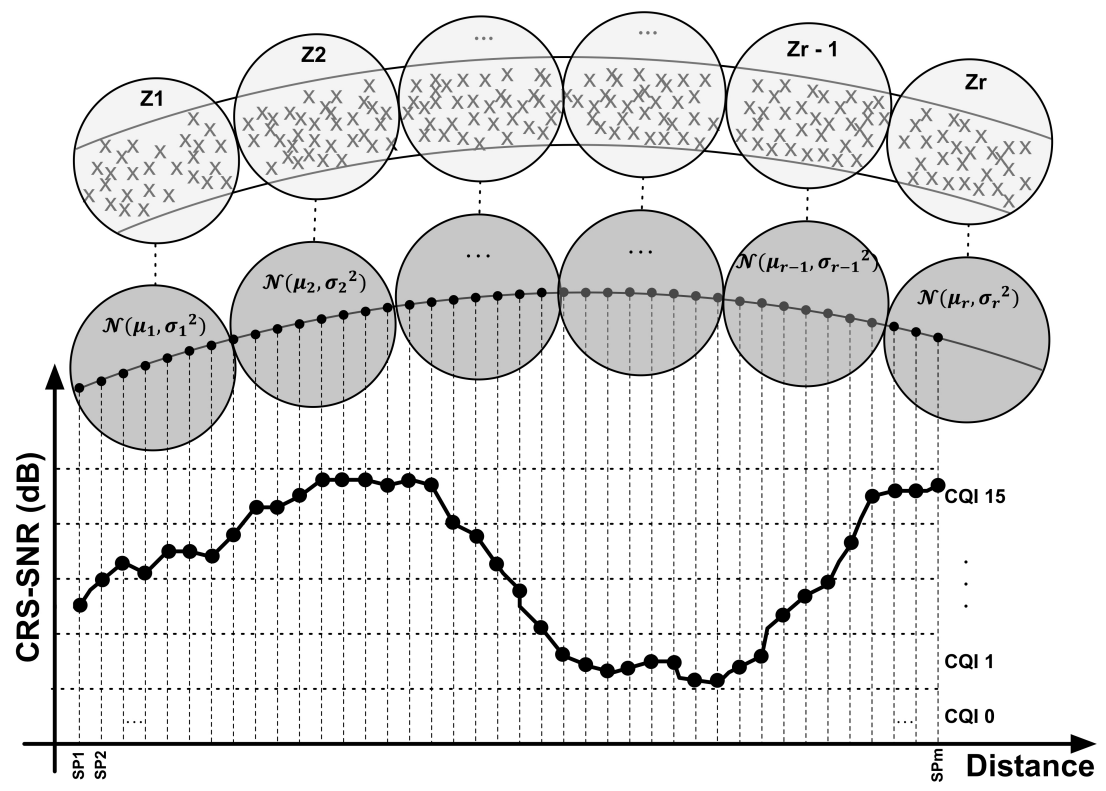

Figure 4: Illustration of the resampling process. Top, zones along the motorway; middle, sampling points within zones whose statistical properties are modeled as normally distributed random variables; bottom, CRS-SNR sampling points and CQI levels along the motorway.

Each one of these samples was then mapped to a corresponding CQI level. The process to map CRS-SNR to CQI is described in the following section. 


\subsection{CRS-SNR to CQI mapping}

In LTE, parameter CQI is reported by the UE to the eNodeB to indicate the quality of the communication channel. These reports are used by the scheduling algorithm at the eNodeB to perform link adaptation through adaptive modulation and coding.

In order to perform link adaptation, the algorithm selects the Modulation and Coding Schemes (MCSs) for the Transport Blocks (TBs) to be allocated to the UE based on its reported CQI. The MCSs are selected to maximize the throughput and to maintain the Block Error Rate (BLER) below the $10 \%$ threshold to comply with the LTE standard [19].

There is a total of 16 CQI levels (0 to 15$)$ defined in the standard, where each level represents a quantized version of the SNR measured at the antenna port of the UE. From the 16 possible levels that parameter CQI can take, 1 and 15 represent the worst and the best channel qualities, respectively. In addition, CQI 0 corresponds to the out-of-range state [19]. In our analysis, it has been considered that a UE reporting CQI 0 cannot support an LTE connection.

In a similar way to CQI, there is a range of 29 MCSs (0 to 28), where each MCS represents a unique combination of modulation and coding rate. Within this range, MCS 0 and MCS 28 correspond to the worst and best combinations (performance-wise), respectively.

Available modulation methods in LTE for data transmission are QPSK, 16QAM and 64QAM. The coding rate, on the other hand, depends on other parameters such as the size of the TB, the number of bits for cyclic redundancy check and the number of resource elements $(R E s)$ available for data transmission [19]. The MCSs and their corresponding modulation methods can be seen in Table 1 [19].

\begin{tabular}{|c|c|c|c|c|c|c|c|c|c|c|c|c|c|c|c|c|c|c|c|c|c|c|c|c|c|c|c|c|c|}
\hline MCS & 0 & 1 & 2 & 3 & 4 & 5 & 6 & 7 & 8 & 9 & 10 & 11 & 12 & 13 & 14 & 15 & 16 & 17 & 18 & 19 & 20 & 21 & 22 & 23 & 24 & 25 & 26 & 27 & 28 \\
\hline Modulation method & & & & & QP & SK & & & & & & & & 16QA & & & & & & & & & $64 \mathrm{Q}$ & $\mathrm{AM}$ & & & & & \\
\hline
\end{tabular}

Table 1: MCSs and modulation methods in LTE [19].

The process that the UE implements to estimate the CQI is not established by the LTE standard and therefore, it is open to chipset manufacturers [6]. Since this process is not clearly defined, we have followed the methodology described in [20] as a reference to estimate the CQI values required by the FSMC. The process to map CRS-SNR values to CQI levels described in [20] is carried out in three steps. In the first step, an averaging window is applied to the CRS-SNR measured at the UE. In the second step, the averaged CRS-SNR is mapped to an MCS. Finally, in the third step, the MCS is converted to a CQI level.

In our analysis, an averaging window of five CRS-SNR measurements was applied in order to reduce variations in the measurements. By using the average CRS-SNR to estimate the CQI, inaccurate MCS allocations are avoided and consequently the BLER is reduced. The average CRS-SNR value was then mapped to an MCS. For this purpose, the modulation curves presented in Figure 5 were used. The modulation curves describe the relation between SNR and BLER for each MCS. 


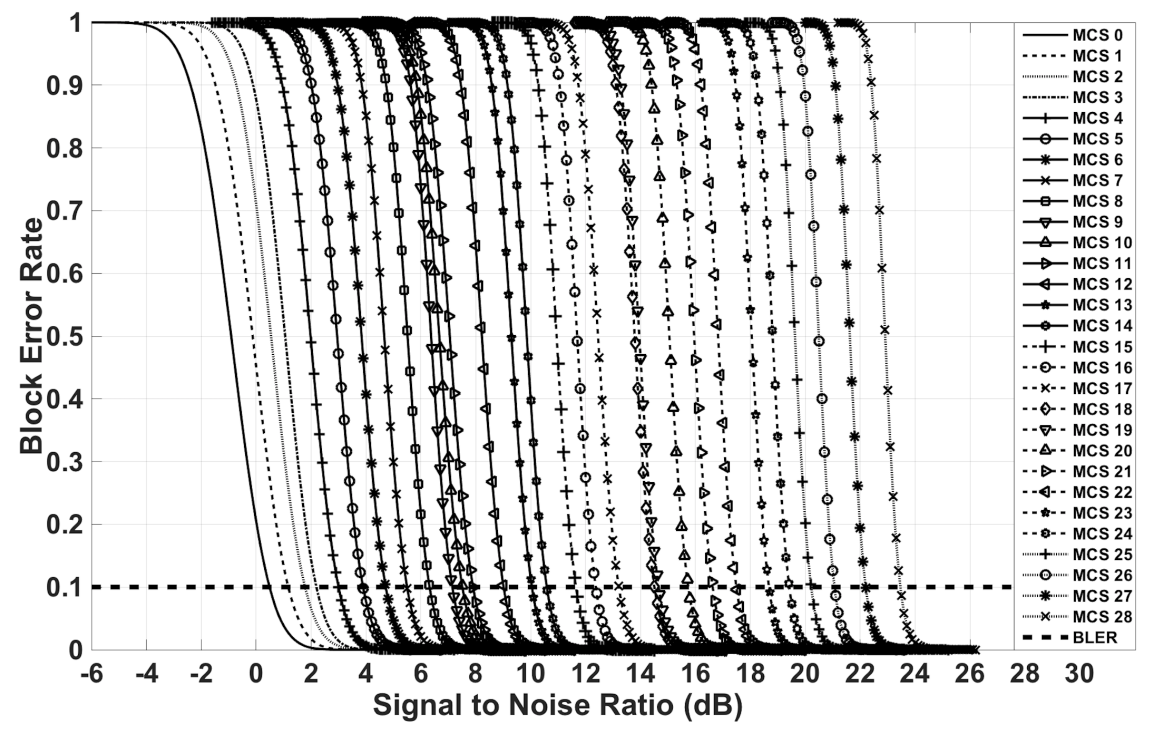

Figure 5: Modulation curves for Modulation and Coding Schemes (MCSs) 0 to 28 and target error rate (10\% of BLER) [20].

By using Figure 5, the average CRS-SNR was mapped to all MCSs and thus, the BLER of each MCS was estimated. From all MCSs, only those with a BLER below the $10 \%$ threshold were considered as viable options. From viable MCSs, the one with the highest performance was chosen to maximize throughput.

Finally, after a specific MCS was obtained, the mapping from MCS to CQI was performed. For this process, the tables found in [20], describing the equivalence between the 29 MCSs and the 16 CQI levels, were used. In cases where the average CRS-SNR was below the SNR threshold for MCS 0, the CRS-SNR was mapped to CQI level 0 and considered as the out-of-range state. This process was repeated for all averaged values of CRS-SNR to obtain their corresponding CQI levels.

\subsection{Markov modeling}

To model the evolution of reported CQI values, a FSMC is proposed. The model is developed under the assumption that the CQI values can be characterized as a discrete random process $X=\left\{X_{m} ; m \in \mathbb{N}\right\}$ that complies with the Markov property. This property establishes that the probability of the system being at a given state $j$ at index $m+1$, is conditioned only on the knowledge that the process is at state $i$ at index $m$, and therefore, independent of all previous states [21].

In this case, to assume that this property holds is equivalent to consider that the space distribution of observed CQI values is such that the probability of measuring a certain CQI value in a nearby location along a route only depends on the value observed at the present location. Note that the chain index in this case represents position instead of time, which is the most frequent case.

For the proposed Markov model, a finite state space $S_{c q i}=\{0,1 \ldots 15\}$ is defined, where each state represents one of the 16 levels of CQI (0 to 15). Moreover, in order to define the transition probability 
between states $i$ and $j$, i.e. $P_{i, j}$, this FSMC is considered to be space-homogeneous, therefore its transition probabilities are independent of the actual value of $m$ and thus, $P_{i, j}$ can be defined as

$$
P_{i, j}=P\left(X_{m+1}=j \mid X_{m}=i\right) ; \forall i, j \in S_{c q i} .
$$

In order to consider the probabilities of all possible transitions between the states of the model, a transition probability matrix $\boldsymbol{P}_{\boldsymbol{c} q \boldsymbol{i}}$ is defined as

$$
\boldsymbol{P}_{\boldsymbol{c q i}}=\left[\begin{array}{ccccc}
P_{0,0} & & \ldots & & P_{0,15} \\
& \ddots & \ddots & \ddots & \\
\vdots & \ddots & P_{i, j} & \ddots & \vdots \\
& \ddots & \ddots & \ddots & \\
P_{15,0} & & \ldots & & P_{15,15}
\end{array}\right] ; i, j \in S_{c q i} .
$$

Recall that the transition probability matrix $\boldsymbol{P}_{\boldsymbol{c q} i}$ is a stochastic matrix, meaning that the total sum of the transition probabilities for state $i$, represented by the $i_{t h}$ row of matrix $\boldsymbol{P}_{\boldsymbol{c q} i}$, must be equal to 1 . Therefore,

$$
\sum_{j=0}^{15} P_{i, j}=\sum_{j=0}^{15} P\left(X_{m+1}=j \mid X_{m}=i\right)=1 ; \forall i \in S_{c q i} .
$$

The transition probability matrix $\boldsymbol{P}_{\boldsymbol{c q} \boldsymbol{i}}$, could also be represented by means of a state transition diagram [22], with vertices representing the states within the finite state space $S_{c q i}$ and edges representing the transition probability $P_{i, j}$ between states $i$ and $j$.

In order to obtain the transition probabilities $P_{i, j}$ from the resampled data, the following procedure was applied. The number of times CQI changed from state $i$ to state $j$ was counted (i.e., $n_{i, j}$ ) when considering two consecutive sampling points along the road (i.e., $m$ and $m+1$ ) and this procedure was repeated for all sampling points depicted in Figure 4. Thus, the set of transition probabilities $P_{i, j}$ were computed as

$$
P_{i, j}=\frac{n_{i, j}}{\sum_{j=0}^{15} n_{i, j}} ; \forall i, j \in S_{c q i}
$$

\section{Results from FSMC modeling and analysis}

The set of transition probabilities were found as described above. The resulting transition probabilities $P_{i, j}$ for all states $i$ and $j$, of the transition probability matrix $\boldsymbol{P}_{\boldsymbol{c q} i \boldsymbol{i}}$, are shown in Table 2 .

From the probability matrix $\boldsymbol{P}_{\boldsymbol{c} q \boldsymbol{i}}$ it can be seen that the use of averaged CRS-SNR measurements causes the CQI levels to remain the same or move only to nearby levels, as expected in a usual situation. As a result of this, the higher values of transition probabilities are the ones close to the main diagonal in Table 2 . 
In contrast, the probability of CQI changing two or more levels from its current value is considerably lower as it can be observed from the transition probabilities outside such main diagonal.

\begin{tabular}{|c|c|c|c|c|c|c|c|c|c|c|c|c|c|c|c|c|}
\hline CQI level & 0 & 1 & 2 & 3 & 4 & 5 & 6 & 7 & 8 & 9 & 10 & 11 & 12 & 13 & 14 & 15 \\
\hline 0 & 0.718 & 0.081 & 0.079 & 0.085 & 0.033 & 0.004 & 0 & 0 & 0 & 0 & 0 & 0 & 0 & 0 & 0 & 0 \\
\hline 1 & 0.24 & 0.144 & 0.194 & 0.244 & 0.139 & 0.039 & 0 & 0 & 0 & 0 & 0 & 0 & 0 & 0 & 0 & 0 \\
\hline 2 & 0.126 & 0.159 & 0.183 & 0.325 & 0.134 & 0.065 & 0.004 & 0.004 & 0 & 0 & 0 & 0 & 0 & 0 & 0 & 0 \\
\hline 3 & 0.054 & 0.048 & 0.077 & 0.384 & 0.253 & 0.155 & 0.023 & 0.006 & 0 & 0 & 0 & 0 & 0 & 0 & 0 & 0 \\
\hline 4 & 0.011 & 0.014 & 0.02 & 0.115 & 0.453 & 0.298 & 0.068 & 0.018 & 0.002 & 0.001 & 0 & 0 & 0 & 0 & 0 & 0 \\
\hline 5 & 0.001 & 0.002 & 0.006 & 0.024 & 0.143 & 0.502 & 0.226 & 0.073 & 0.022 & 0.001 & 0 & 0 & 0 & 0 & 0 & 0 \\
\hline 6 & 0 & 0.001 & 0.001 & 0.005 & 0.047 & 0.175 & 0.485 & 0.192 & 0.089 & 0.004 & 0.001 & 0 & 0 & 0 & 0 & 0 \\
\hline 7 & 0 & 0 & 0 & 0.001 & 0.011 & 0.058 & 0.153 & 0.534 & 0.224 & 0.015 & 0.004 & 0 & 0 & 0 & 0 & 0 \\
\hline 8 & 0 & 0 & 0 & 0 & 0.001 & 0.006 & 0.039 & 0.23 & 0.542 & 0.135 & 0.043 & 0.004 & 0 & 0 & 0 & 0 \\
\hline 9 & 0 & 0 & 0 & 0 & 0.001 & 0.001 & 0.009 & 0.084 & 0.227 & 0.505 & 0.144 & 0.027 & 0.002 & 0 & 0 & 0 \\
\hline 10 & 0 & 0 & 0 & 0 & 0 & 0 & 0.001 & 0.011 & 0.05 & 0.397 & 0.387 & 0.133 & 0.016 & 0.005 & 0 & 0 \\
\hline 11 & 0 & 0 & 0 & 0 & 0 & 0 & 0 & 0.004 & 0.011 & 0.225 & 0.17 & 0.463 & 0.094 & 0.029 & 0.004 & 0 \\
\hline 12 & 0 & 0 & 0 & 0 & 0 & 0 & 0 & 0 & 0 & 0.07 & 0.108 & 0.338 & 0.39 & 0.061 & 0.019 & 0.014 \\
\hline 13 & 0 & 0 & 0 & 0 & 0 & 0 & 0 & 0 & 0 & 0 & 0.071 & 0.153 & 0.294 & 0.4 & 0.047 & 0.035 \\
\hline 14 & 0 & 0 & 0 & 0 & 0 & 0 & 0 & 0 & 0 & 0 & 0 & 0 & 0.333 & 0.381 & 0.286 & 0 \\
\hline 15 & 0 & 0 & 0 & 0 & 0 & 0 & 0 & 0 & 0 & 0 & 0 & 0 & 0.111 & 0.111 & 0.445 & 0.333 \\
\hline
\end{tabular}

Table 2: Transition probability matrix $\boldsymbol{P}_{\boldsymbol{c q} \boldsymbol{i}}$.

As an example of the information provided in matrix $\boldsymbol{P}_{\boldsymbol{c q} \boldsymbol{i}}$, it can be mentioned that the first row presents the transition probabilities for CQI level 0, which is used by the UE to report the out-of-coverage state. The value of 0.718 for CQI level 0 means that a UE without LTE coverage is more likely to remain in the same state in the next sampling point.

Furthermore, it is interesting to note that some transition probabilities in the main diagonal, i.e. $P_{i, i}$, are significantly higher than the other probabilities in the same row. Thus, the CQI value is very likely to remain in the same value for the next sampling point. This is more noticeable for CQI levels 4 through 9 , meaning that a UE is more likely to have a stable LTE connection when reaching these CQI levels.

By observing the characteristics of the transition probability matrix $\boldsymbol{P}_{\boldsymbol{c q} \boldsymbol{i}}$, it can also be inferred that the process can move back and forth among all states belonging to $S_{c q i}$ in an arbitrary (random) number of steps. Therefore, it can be concluded that the FSMC is irreducible and its steady state probabilities can be calculated [22]. The steady state probabilities for the transition probability matrix $\boldsymbol{P}_{\boldsymbol{c q} \boldsymbol{i}}$ can be calculated through

$$
\boldsymbol{\pi}_{\boldsymbol{c q} \boldsymbol{i}}=\lim \left(\boldsymbol{P}_{\boldsymbol{c q} \boldsymbol{i}}\right)^{n} \text { when } n \rightarrow \infty \text {. }
$$

For a large enough $n$, the results are presented in Table 3 . 


\begin{tabular}{ccccccccccccccccc}
\hline CQI level & $\mathbf{0}$ & $\mathbf{1}$ & $\mathbf{2}$ & $\mathbf{3}$ & $\mathbf{4}$ & $\mathbf{5}$ & $\mathbf{6}$ & $\mathbf{7}$ & $\mathbf{8}$ & $\mathbf{9}$ & $\mathbf{1 0}$ & $\mathbf{1 1}$ & $\mathbf{1 2}$ & $\mathbf{1 3}$ & $\mathbf{1 4}$ & $\mathbf{1 5}$ \\
\hline Probability & 0.018 & 0.007 & 0.009 & 0.029 & 0.069 & 0.129 & 0.144 & 0.199 & 0.193 & 0.114 & 0.051 & 0.026 & 0.008 & 0.003 & 0.001 & 0 \\
\hline
\end{tabular}

Table 3: Steady state probability vector $\boldsymbol{\pi}_{\boldsymbol{c q} \boldsymbol{i}}$.

In the steady state probability vector $\boldsymbol{\pi}_{c q i}$, the probability for CQI level 0 is particularly relevant. As mentioned above, CQI 0 is reported by the UE to indicate the out-of-coverage state. Therefore, the value for the steady probability of this level could be interpreted as the outage probability of the LTE connection. In the analyzed scenario, this accounts for $1.8 \%$.

The steady state probability values for the upper levels of CQI can also be observed in Table 3. For CQI levels 10 through 15, the total probability is around $9 \%$. This would mean that there is less than $10 \%$ probability that a UE can achieve the highest levels of throughput in the downlink. This is a logical outcome, since these levels are only achieved when a UE is really close to the eNodeB and the propagation conditions are ideal in terms of noise and interference.

It is also worth pointing out that the steady state probability values for CQI levels 4 through 9 account for around $85 \%$ of the total probability. This result suggests that most of the coverage along the motorway is good enough to maintain stable LTE connections.

For the sake of clarity, the whole methodology that was followed for processing the collected data is summarized by means of the flowchart shown in Figure 6. In the section to follow, a theoretical model to compute the downlink transmission capacity is presented. Such model is then used in combination with the transition probabilities of the CQI FSMC shown in Table 2 to present a case study that estimates the transmission capacity in the analyzed scenario.
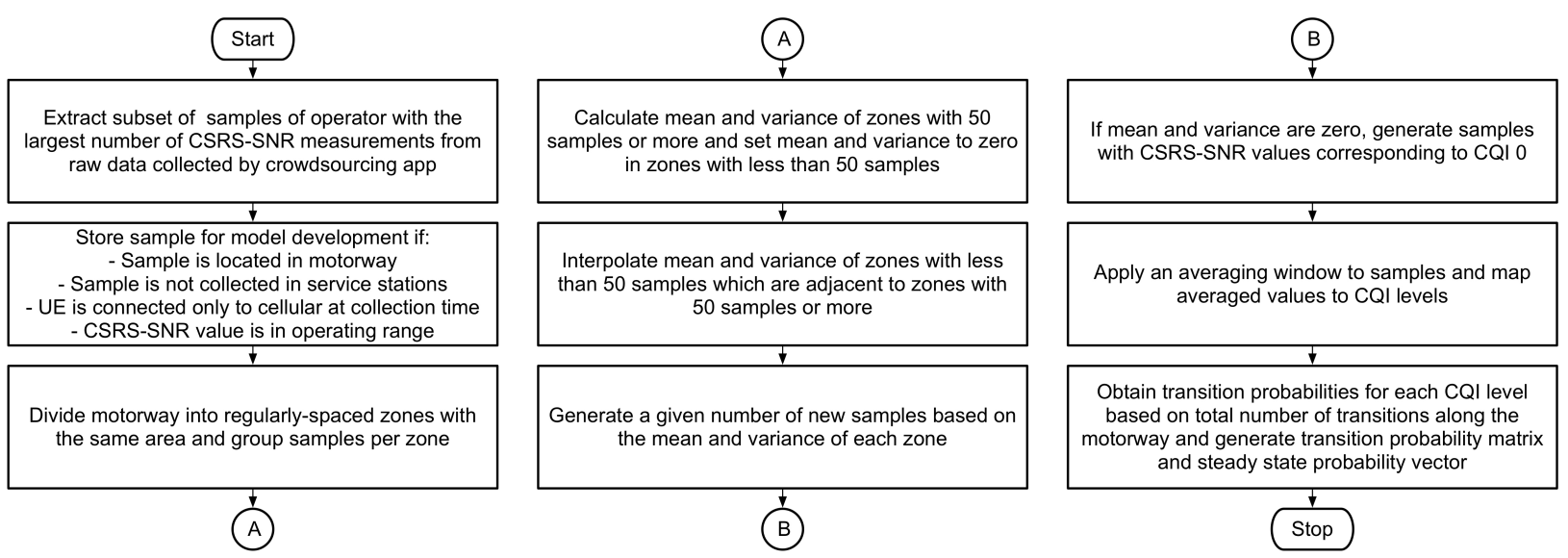

Figure 6: Modeling-methodology flowchart. 


\section{Downlink transmission capacity model}

In this section, a theoretical model for estimating the transmission capacity of the LTE downlink channel is described. The purpose of this model is twofold. On one hand, it allows us to obtain the maximum transmission capacity of the downlink channel in the analyzed scenario. On the other hand, the model illustrates how the FSMC can be used for benchmarking resource allocation algorithms, in scenarios where V2I networks could be deployed in the near future.

In order to properly describe the proposed model, in what follows we provide an overview of some required concepts related to channel estimation and adaptive modulation and coding.

\subsection{Channel estimation and adaptive modulation and coding}

LTE makes use of adaptive modulation and coding. That is, the scheduling algorithm at the eNodeB dynamically adapts the modulation and coding rate based on channel estimations performed by the UE. As a result of this, the data rate continuously changes depending on the quality of the channel experienced by the UE.

The UE estimates the quality of the communication channel by measuring the Signal to Noise Ratio (SNR) of the received signal. Then, the estimated channel quality is reported to the scheduling algorithm at the eNodeB in the form of a CQI level.

On the eNodeB side, the scheduling algorithm uses the reported values of CQI to adapt the modulation and coding rate for the Resource Blocks (RBs) to be granted to the reporting UE.

The selection of the adequate combination of modulation and coding rate can be based on a combination of the reported CQI, the category of the reporting UE, specific quality of service policies and available resources at the moment of either resource request or allocation [23]. From all these factors, parameter CQI is the only one that quantifies the quality of the communication channel.

Available modulation methods in LTE for data transmission are QPSK, 16QAM and 64QAM and each method supports the transmission of 2, 4 and 6 bits per OFDM symbol (as explained below an OFDM symbol is closely related to an $R E$ ), respectively. In the model, the modulation methods were associated to each CQI level based on Table 4.

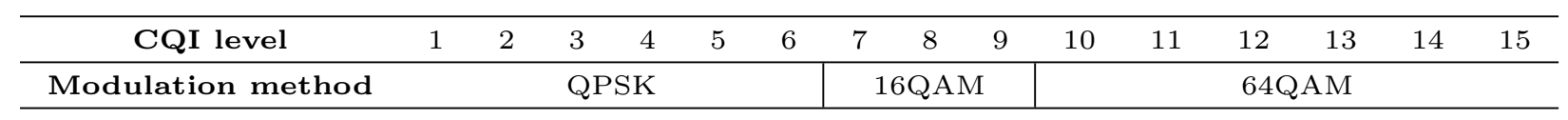

Table 4: CQI levels and modulation methods [19].

In addition, the coding rate is established based on a combination of a number of parameters. These parameters are: the number of bits required for Cyclic Redundancy Check $(C R C)$, the Transport Block Size (TBS), the number of REs assigned to the Physical Downlink Shared Channel (PDSCH) and the 
number of bits that can be transmitted per resource element $\left(B_{R E}\right)[19]$. In the model, the coding rates were associated to each CQI level based on Table 5.

\begin{tabular}{cccccccccccccccc}
\hline CQI level & 1 & 2 & 3 & 4 & 5 & 6 & 7 & 8 & 9 & 10 & 11 & 12 & 13 & 14 & 15 \\
\hline Code_rate per 1024 & 78 & 120 & 193 & 308 & 449 & 602 & 378 & 490 & 616 & 466 & 567 & 666 & 772 & 873 & 948 \\
\hline
\end{tabular}

Table 5: CQI levels and coding rates, based on [19].

As previously mentioned, the goal of this section is to estimate the transmission capacity of the downlink channel. For this reason, the model focuses on $P D S C H$, which is used by the eNodeB for transmission of dedicated traffic to UEs. A detailed analysis to estimate the amount of resources that can be allocated to $P D S C H$ is presented in the sections to follow.

\subsection{Theoretical model definition}

The model is intended to obtain the maximum transmission capacity of the downlink channel and was obtained by computing the resources available for $P D S C H$, which is used in LTE for transmitting user information. The resources are considered in terms of $R E s$, which are the smallest resource units in LTE and each one of them is composed of one $15 \mathrm{kHz}$ subcarrier and one OFDM symbol.

The number of available $R E s$ for $P D S C H$ is denoted as $P D S C H_{R E}$. To compute this quantity, the number of $R E s$ allocated to all control channels in the downlink $\left(D C C H_{R E}\right)$ are subtracted from the total number of $R E s$ allocated to the entire downlink channel $\left(T D_{R E}\right)$. Therefore, on a frame basis, parameter $P D S C H_{R E}$ can be computed as

$$
P D S C H_{R E}=T D_{R E}-D C C H_{R E} .
$$

\subsection{1. $T D_{R E}$ computation}

For the computation of $T D_{R E}$, resources have to be considered in both frequency and time.

Regarding frequency resources, the channel bandwidth in terms of the number of Resource Blocks $(R B s)$ was considered. In LTE, the following six different channel bandwidth configurations are available: 1.4, 3, 5, 10, 15 and $20 \mathrm{MHz}$. This bandwidth configurations support a total of 6, 15, 25, 50, 75 and $100 \mathrm{RBs}$, respectively. The $R B$ is the smallest allocatable unit in LTE and is composed of twelve $15 \mathrm{kHz}$ subcarriers and up to 7 OFDM symbols.

As for time resources, the Cycle Prefix $(C P)$ version was considered. It is worth pointing out that there is a normal and an extended version of $C P$. The normal version sets the size of each $R B$ to 7 OFDM symbols ( $R B_{\text {NORMAL }}$ ), whereas the extended version sets the size of each $R B$ to 6 OFDM symbols (RB EXTENDED $)$.

After frequency and time resources are defined, the transmission mode has to be selected between Time Division Duplex (TDD) and Frequency Division Duplex (FDD). For the model, TDD was chosen as the 
transmission mode. The reason for selecting TDD over FDD is that TDD allows us to select the proportion of resources to be allocated between downlink and uplink transmissions not only using a $10 \mathrm{~ms}$ frame, but also down to a $1 \mathrm{~ms}$ subframe. Thus, it allows a more precise selection of model parameters.

By selecting TDD, seven frame configurations can be chosen. Each configuration defines a different ratio of the number of subframes for downlink $\left(D L_{S F}\right)$ to the number of uplink subframes $\left(U P_{S F}\right)$ within a frame. As the proposed model computes the available resources for the downlink, only $D L_{S F}$ was considered.

TDD also requires the implementation of a Special Subframe $(S S)$ for switching between downlink and uplink transmissions. Within the $S S$, there is a portion of OFDM symbols used for downlink $\left(D W_{P T S}\right)$, uplink $\left(U P_{P T S}\right)$ and a guard period $(G P)$. TDD supports 9 and $7 S S$ configurations for the normal and extended version of $C P$, respectively. Given that the model is for downlink transmissions, only $D W_{P T S}$ symbols were considered.

The total number of $R E s$ allocated to the downlink channel on a frame basis $T D_{R E}$ was computed through

$$
T D_{R E}=R B \times\left(\left(D L_{S F} \times R B_{N O R M A L} \times 2\right)+D W_{P T S}\right) \times 12,
$$

where $R B_{\text {NORMAL }}$ was selected to allocate an additional OFDM symbol per subframe. Moreover, in Eq. (10), numbers 2 and 12 come from considering 2 RBs per subframe and 12 subcarriers per RB, respectively.

\subsection{2. $D C C H_{R E}$ computation}

The computation of $D C C H_{R E}$ was performed by taking into account the number of $R E s$ required by each control channel at the physical layer.

To this end, the following physical downlink control channels were considered: Physical Downlink Control Channel $(P D C C H)$, Physical Broadcast Channel $(P B C H)$, Physical Control Format Indicator Channel $(P C F I C H)$ and Physical Hybrid ARQ Indicator Channel (PHICH). In addition to these channels, Primary Synchronization Signals (PSS), Secondary Synchronization Signals $(S S S)$ and $C R S$ were also considered.

The number of $R E s$ required by the $P D C C H$ on a frame basis is defined as $P D C C H_{R E}$ and can be obtained as

$$
P D C C H_{R E}=R B \times\left(C F I \times\left(D L_{S F}+S S\right)\right) \times 12 .
$$

In Eq. (11), CFI stands for Control Format Indicator. This parameter establishes that, in time domain, the number of OFDM symbols destined to the PDCCH can be 2,3 and 4 for $1.4 \mathrm{MHz}$, whereas they can be 1, 2 and 3 for higher bandwidth configurations. In the frequency domain, the PDCCH extends along the entire channel independently of the bandwidth configuration.

The number of REs required by the $P B C H$ is defined as $P B C H_{R E}$. This parameter has a fixed value of $240 R E s$ independently of the bandwidth configuration and it is transmitted every 4 frames. Therefore, in the model, $P B C H_{R E}$ was set to 240 , on a frame basis. 
Parameters PCFICH and PHICH were also considered in the model. However, the REs required by these channels overlap with $P D C C H_{R E}$, and thus are already considered in Eq. (11).

Regarding $C R S$, the number of $R E s$ on a frame basis is defined as $C R S_{R E}$ and was obtained through

$$
C R S_{R E}=R B \times\left(D L_{S F}+S S\right) \times N \times 8 \times 3 / 4
$$

where $N$ represents the number of antennas at the eNodeB. For the model, $N$ was set to 1 (SISO mode). Moreover, numbers 8 and $3 / 4$ represent the number of $C R S$ per $R B$ and an overlapping factor, respectively. This factor was introduced to take into account the $1 / 4$ of $R E$ that overlaps with the $P D C C H_{R E}$.

Finally, the number of $R E s$ for $P S S$ and $S S S$ were also analyzed. However, these $R E s$, required by these signals, overlap with $P D C C H_{R E}$ in a similar way as $P C F I C H$ and $P H I C H$, and thus, were already considered in Eq. (11).

Having established the number of $R E s$ required by each control channel at the physical layer, $D C C H_{R E}$ was computed, on a frame basis, by

$$
D C C H_{R E}=P D C C H_{R E}+P B C H_{R E}+C R S_{R E}
$$

In Eq. (13), the REs for PCFICH, PHICH,PSS and SSS are not shown since they were already considered in Eq. (11) for $P D C C H_{R E}$.

\subsubsection{Downlink transmission capacity computation}

Having defined the computation of $T D_{R E}$ and $D C C H_{R E}$ in (10) and (13) respectively, $P D S C H_{R E}$ can be computed through Eq. (9) on a frame basis.

The obtained $P D S C H_{R E}$ can then be used to compute the downlink transmission capacity in bits per second $\left(D T C_{b p s}\right)$ through

$$
D T C_{b p s}=P D S C H_{R E} \times B_{R E} \times C R \times 1 /(F D) .
$$

In Eq. (14), $B_{R E}$ is the number of bits that can be transmitted per $R E, C R$ is the coding rate and $F D$ represents the frame duration, i.e. $10 \mathrm{~ms}$.

\section{Downlink transmission capacity results}

In this section, the LTE downlink transmission capacity is obtained for the M1 motorway based on the CQI levels generated by the FSMC. As stated before, this analysis is presented to illustrate how the results from the FSMC could be used for benchmarking resource allocation algorithms in vehicular environments.

For this purpose, the model described by Eq. (14) was combined with the CQI levels generated by the FSMC through the transition probabilities shown in Table 2. For the model defined by (14), $P D S C H_{R E}$ 
was obtained based on Eq. (9) and the configuration parameters summarized in Table 6, whereas $B_{R E}$ and $C R$ were obtained based on the generated CQI levels and the values from Table 4 and Table 5, respectively.

\begin{tabular}{lr}
\hline Parameter & Value \\
\hline Channel bandwidth $(\mathrm{MHz})$ & $5,10,20$ \\
$C P$ version & Normal \\
Transmission mode & TDD \\
TDD frame configurations & $1,3,5$ \\
Special subframe configuration & 1 \\
$P D C C H$ size (ODFM symbols) & 1 \\
Number of transmission and reception antennas & 1 (SISO) \\
\hline
\end{tabular}

Table 6: Selected configuration parameters.

Based on the configuration parameters shown in Table 6, the minimum, maximum and mean downlink transmission capacity values in $\mathrm{Mb} / \mathrm{s}$ were obtained for the analyzed scenario. These results are summarized in Table 7 .

\begin{tabular}{|c|c|c|c|c|c|}
\hline Channel bandwidth (MHz) & TDD Configuration & SS Configuration & $\operatorname{Min}(\mathrm{Mb} / \mathrm{s})$ & $\operatorname{Max}(\mathrm{Mb} / \mathrm{s})$ & Mean $(\mathrm{Mb} / \mathrm{s})$ \\
\hline 5 & 1 & 1 & 0.248 & 9.056 & 2.572 \\
\hline 5 & 3 & 1 & 0.369 & 13.472 & 3.833 \\
\hline 5 & 5 & 1 & 0.483 & 17.638 & 5.017 \\
\hline 10 & 1 & 1 & 0.504 & 18.388 & 5.222 \\
\hline 10 & 3 & 1 & 0.746 & 27.220 & 7.735 \\
\hline 10 & 5 & 1 & 0.975 & 35.552 & 10.104 \\
\hline 20 & 1 & 1 & 1.016 & 37.052 & 10.533 \\
\hline 20 & 3 & 1 & 1.500 & 54.715 & 15.545 \\
\hline 20 & 5 & 1 & 1.957 & 71.38 & 20.283 \\
\hline
\end{tabular}

Table 7: Downlink transmission capacity for configuration parameters in Table 6 .

In Table 7, it can be seen that for the same bandwidth e.g. $10 \mathrm{MHz}$, the mean transmission capacity approximately varies in steps of $2.5 \mathrm{Mb} / \mathrm{s}$ among the selected TDD configurations. This occurs due to the fact that the number of subframes allocated to the downlink direction increases from 4 for TDD configuration 1 , to 6 and 8 for TDD configurations 3 and 5, respectively. Moreover, these results imply that the selected TDD configuration considerably impacts the final downlink transmission capacity.

Furthermore, Table 7 shows that, for the selected configuration parameters, the maximum transmission capacity that can be achieved is approximately $71 \mathrm{Mb} / \mathrm{s}$. This peak value is achieved when the the entire channel bandwidth, i.e. $20 \mathrm{MHz}$, is allocated and the best channel quality, i.e. CQI 15, is reported. However, scenarios where values close to this peak can be achieved are unlikely to occur and are only presented to show the maximum capacity that can be estimated by the model based on the CQI levels from the FSMC.

In order to perform a more detailed analysis, a subset of configuration parameters was selected. This subset of parameters is presented in Table 8. The rest of the configuration parameters are the same as the 
ones previously presented in Table 6.

\begin{tabular}{lr}
\hline Parameter & Value \\
\hline Channel bandwidth $(\mathrm{MHz})$ & 10 \\
TDD frame configuration & 3 \\
Special subframe configuration & 1 \\
\hline
\end{tabular}

Table 8: Subset of configuration parameters.

Based on the subset of configuration parameters presented in Table 8, the downlink transmission capacity in $\mathrm{Mb} / \mathrm{s}$ was analyzed. For this purpose, Figure 7 shows the probability mass function for the downlink transmission capacity.

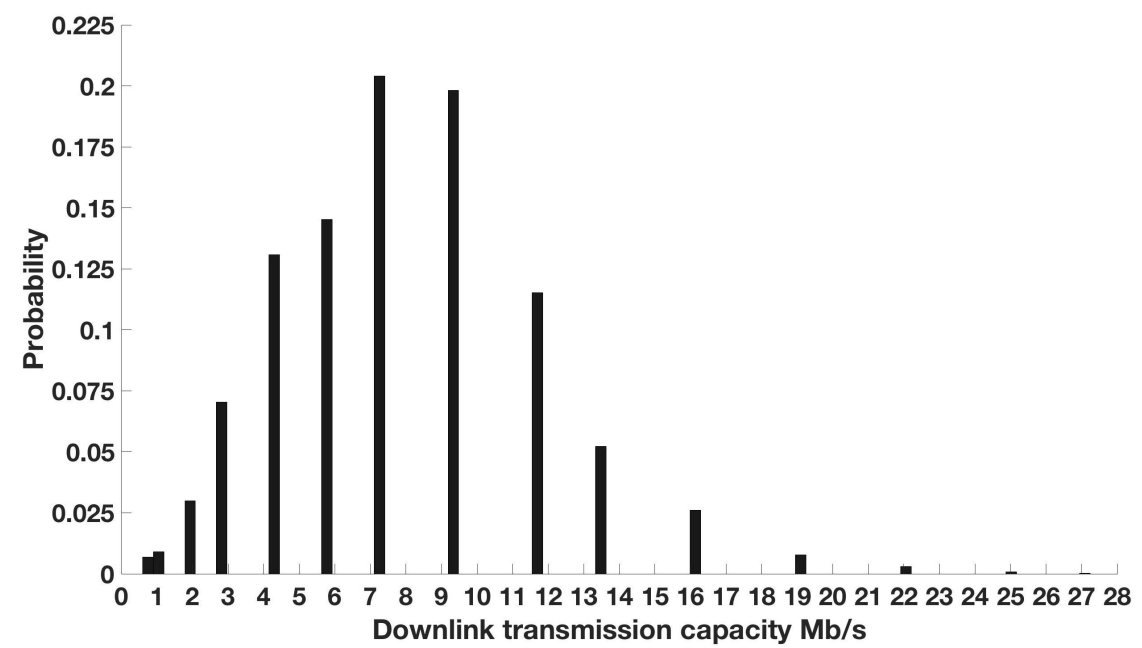

Figure 7: Probability distribution for downlink transmission capacity and configuration parameters in Table 8.

In Figure 7, a total of 15 values corresponding to the capacity that can be achieved by each of the 15 levels of CQI can be observed, where the leftmost and rightmost values correspond to CQI 1 and 15, respectively, whereas CQI 0 is not shown, since it represents the out-of-range state and therefore, it was not considered in the computation.

From Figure 7, the mean transmission capacity is $7.735 \mathrm{Mb} / \mathrm{s}$ (as can be seen in Table 7 for the corresponding configuration parameters). This value is considerably low compared with approximately $27 \mathrm{Mb} / \mathrm{s}$ that can be achieved when CQI level 15 is in use, as can be seen in the rightmost part of Figure 7. This relatively low mean transmission capacity should be expected in high mobility vehicular environments, where the channel quality experienced by UEs must be worse in comparison with the static case.

Another consequence of the low channel quality in the analyzed scenario is the low probability of achieving a higher transmission capacity. This can also be seen in Figure 7, where the probability for achieving a transmission capacity above $16 \mathrm{Mb} / \mathrm{s}$ is almost negligible in comparison with lower values.

In addition, statistics regarding the modulation methods that are utilized along the motorway were also 
obtained. It turns out that utilization percentages for QPSK, 16QAM and 64QAM are 39\%, 52\% and 9\%, respectively.

These results suggest that, as a consequence of the channel quality conditions in the analyzed scenario, the resource allocation algorithm implements the lowest modulation methods, i.e. QPSK and 16QAM, along $91 \%$ of the motorway in order to maintain the BLER below the $10 \%$ threshold. However, by implementing the lowest modulation methods, the downlink transmission capacity is considerably reduced as observed from the mean transmission capacity values presented in Table 7 .

For the sake of clarity, the whole process to estimate the downlink transmission capacity in the analyzed scenario is summarized by means of the flowchart shown in Figure 8.
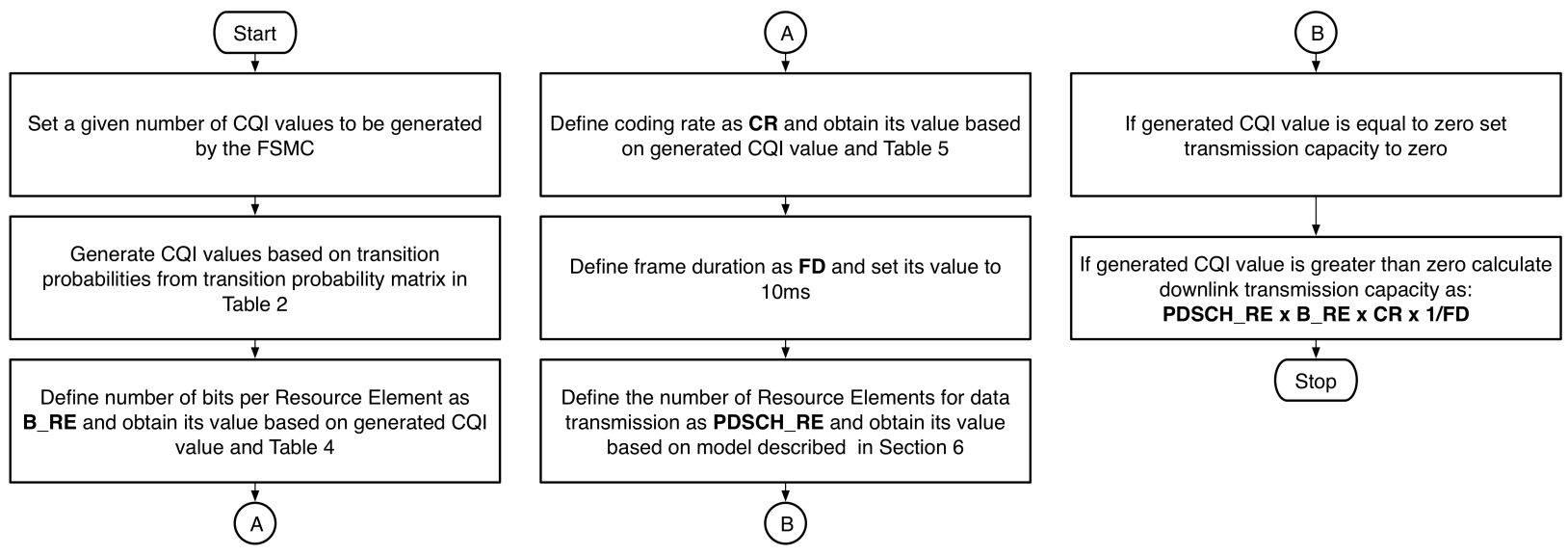

Figure 8: Downlink transmission capacity estimation flowchart.

In addition to this case study, the section to follow discusses other potential applications, as well as the limitations of the CQI-FSMC.

\section{Uses and limitations of the CQI FSMC}

\subsection{Potential applications of the model}

It can be recalled that the main goal of the proposed model is to contribute with the means required for benchmarking resource allocation algorithms in LTE for V2I communications. To do this, the model provides a fairly straightforward way of generating CQI reports with similar statistical properties to those generated by UEs in a vehicular environment.

As examples of algorithms that can be evaluated with this approach, we can mention Best CQI [24] and Proportional Fair [24]. These algorithms have different aims, Best CQI is intended to maximize throughput, whereas Proportional Fair tries to maintain an adequate balance between throughput and fairness. However, in both cases, a sequence of generated CQI reports from the model would allow us to assess their performance in vehicular applications. 
Besides benchmarking of resource allocation schemes, a different application of the model could be analysis of link reliability for safety applications in V2I communications. In this case, reliability can be seen as the probability of CQI reports being above the lowest thresholds, e.g. CQI 2 or CQI 3, where link connectivity is prioritized over transmission capacity. The results would allow to analyze the probability of maintaining a V2I connection during a consecutive number of CQI reports, which is critical for safety applications in vehicular networks.

Another application worth mentioning is the estimation of the expected level of availability of link capacity for infotainment applications in V2I communications. For this type of applications, availability could be related to the probability of CQI reports being above a threshold that guarantees a minimum downlink channel capacity for a given number of consecutive reports. This information is relevant for deploying resource-intensive infotainment applications.

\subsection{Limitations of the model}

The data provided by the crowdsourcing application OpenSignal (i.e., to create experimental maps of cell coverage) up to a large extent matched with what was required by our study. However, since the application was not custom-made for our purposes, some desirable features for our study were not available in the data set. For instance, there were some surveyed areas that did not have an amount of samples large enough to fit a probabilistic model, so that some procedures, described above, had to be applied, where possible, in order to estimate the statistical distribution of samples in these cases. Another issue was that information regarding the speed of the vehicle was not included in the records, so that we had to make use of some heuristics in order to reasonably determine when the samples were collected by moving UEs.

The effect of these factors, just mentioned, on the accuracy of the model cannot be overlooked. A decrease in accuracy can arise, for instance, when no LTE coverage is assumed in the motorway sections which had a low number of samples. Another limitation of the model is that it does not capture different levels of mobility and their effect on both channel quality and system performance. This study is not possible without information regarding the speed of the vehicles. Therefore, the consequences of these limitations on the accuracy of the model have to be taken into account prior to its use in applications such as the ones described above.

\section{Conclusions}

This paper introduces a model intended to capture the statistical behavior of the Long-Term Evolution downlink channel quality for use in studies related to vehicle-to-infrastructure communications. It makes use of a Markov chain in order to represent how the channel quality indicator levels change from point to 
point along a motorway. The model is flexible enough to be used in both theoretical analysis and simulationbased studies. Although it was developed from measurements corresponding to a specific time and location, it can be used to represent similar scenarios to the one herein described.

The proposed model was derived from the analysis of a large number of measurements, which were obtained by using the crowdsourcing-based approach. That is, measurements were recorded and contributed by real users driving along a motorway. As it is discussed in this paper, this approach has several advantages when compared to more traditional surveying techniques. In brief, since all users that download and use a crowdsourcing application in their smartphones become sensor nodes, this data collection strategy becomes highly cost-effective. Furthermore, crowdsourced data came from heterogeneous devices under diverse situations, so that this approach allowed us to observe the performance of LTE connectivity in real-world situations. However, great care has to be put into making the readings suitable for modeling and analysis. To this end, we present a methodology, which can be replicated by other works to make use of crowdsourced data.

We also discussed various potential applications of the proposed model such as benchmarking of scheduling algorithms and evaluation of performance indicators for safety and infotainment applications in vehicleto-infrastructure communications.

\section{Acknowledgments}

This work was supported by the British Council Newton Institution Links Programme IL 172728189; UNAM-DGAPA PAPIIT IN116316; and CONACyT PEI 230023.

Authors also thank OpenSignal for providing the dataset used in our analysis.

\section{References}

[1] G. Araniti, C. Campolo, M. Condoluci, A. Iera, A. Molinaro, LTE for vehicular networking: a survey, IEEE Communications Magazine 51 (5) (2013) 148-157. doi:10.1109/MCOM.2013.6515060.

[2] Z. Hameed Mir, F. Filali, LTE and IEEE 802.11p for vehicular networking: a performance evaluation, EURASIP Journal on Wireless Communications and Networking 2014 (1) (2014) 1-15. doi:10.1186/1687-1499-2014-89.

[3] E. Dahlman, S. Parkvall, J. Skld, Chapter 10 - downlink physical-layer processing, in: E. Dahlman, , S. Parkvall, , J. Skld (Eds.), 4G LTE/LTE-Advanced for Mobile Broadband, Academic Press, Oxford, 2011, pp. 143 - 202. doi:https://doi.org/10.1016/B978-0-12-385489-6.00010-2.

[4] A. Elnashar, M. A. El-Saidny, Looking at LTE in practice: A performance analysis of the LTE system based on field test results, IEEE Vehicular Technology Magazine 8 (3) (2013) 81-92. doi:10.1109/MVT.2013.2268334.

[5] V. Sevindik, J. Wang, O. Bayat, J. Weitzen, Performance evaluation of a real long term evolution (LTE) network, in: 37th Annual IEEE Conference on Local Computer Networks - Workshops, 2012, pp. 679-685. doi:10.1109/LCNW.2012.6424050.

[6] S. Avallone, N. Pasquino, S. Zinno, D. Casillo, Smartphone-based measurements of LTE network performance, in: 2017 IEEE International Instrumentation and Measurement Technology Conference (I2MTC), 2017, pp. 1-6. doi:10.1109/I2MTC.2017.7969854. 
[7] L. C. Gimenez, M. C. Cascino, M. Stefan, K. I. Pedersen, A. F. Cattoni, Mobility performance in slow- and highspeed LTE real scenarios, in: 2016 IEEE 83rd Vehicular Technology Conference (VTC Spring), 2016, pp. 1-5. doi:10.1109/VTCSpring.2016.7504347.

[8] H.-Y. Kim, D.-M. Kang, J.-H. Lee, T.-M. Chung, A performance evaluation of cellular network suitability for VANET, International Journal of Electrical, Computer, Energetic, Electronic and Communication Engineering 6 (4) (2012) 448 451.

[9] J. H. Schaffner, H. J. Song, A. Bekaryan, T. Talty, D. Carper, E. Yasan, Scanner based drive test LTE capacity measurements with MIMO antennas placed inside the vehicle, in: 2015 IEEE International Conference on Microwaves, Communications, Antennas and Electronic Systems (COMCAS), 2015, pp. 1-4. doi:10.1109/COMCAS.2015.7360365.

[10] I. Rodriguez, E. P. L. Almeida, M. Lauridsen, D. A. Wassie, L. C. Gimenez, H. C. Nguyen, T. B. Soerensen, P. Mogensen, Measurement-based evaluation of the impact of large vehicle shadowing on V2X communications, in: European Wireless 2016; 22th European Wireless Conference, 2016, pp. 1-8.

[11] W. Li, R. K. P. Mok, D. Wu, R. K. C. Chang, On the accuracy of smartphone-based mobile network measurement, in: 2015 IEEE Conference on Computer Communications (INFOCOM), 2015, pp. 370-378. doi:10.1109/INFOCOM.2015.7218402.

[12] Y. Xu, Z. Wang, W. K. Leong, B. Leong, An end-to-end measurement study of modern cellular data networks, in: M. Faloutsos, A. Kuzmanovic (Eds.), Passive and Active Measurement, Springer International Publishing, Cham, 2014, pp. $34-45$.

[13] S. Sonntag, L. Schulte, J. Manner, Mobile network measurements - it's not all about signal strength, in: 2013 IEEE Wireless Communications and Networking Conference (WCNC), 2013, pp. 4624-4629. doi:10.1109/WCNC.2013.6555324.

[14] J. Cainey, B. Gill, S. Johnston, J. Robinson, S. Westwood, Modelling download throughput of LTE networks, in: 39th Annual IEEE Conference on Local Computer Networks Workshops, 2014, pp. 623-628. doi:10.1109/LCNW.2014.6927712.

[15] Openstreetmap, accessed: 2017-11-27.

URL http://www.openstreetmap.org/

[16] Motorway 1 services, accessed: 2017-11-27. URL https://motorwayservicesonline.co.uk/M1

[17] R. Jain, The Art of Computer Systems Performance Analysis: Techniques for Experimental Design, Measurement, Simulation, and Modeling, Wiley, 1990.

[18] A. Leon-Garcia, Probability, Statistics, and Random Processes for Electrical Engineering, Pearson/Prentice Hall, 2008.

[19] 3GPP, Evolved Universal Terrestrial Radio Access (E-UTRA); Physical Layer Procedures, TS 36.213 V8.8.0, 3rd Generation Partnership Project (3GPP) (2009).

[20] Riverbed, Riverved Modeler version 18.5 (2017).

URL https://www.riverbed.com

[21] E. Çinlar, Introduction to Stochastic Processes, Dover Books on Mathematics Series, Dover Publications, Incorporated, 2013.

[22] G. Bolch, S. Greiner, H. de Meer, K. Trivedi, Queueing Networks and Markov Chains: Modeling and Performance Evaluation with Computer Science Applications, Wiley, 2006.

[23] S. Sesia, I. Toufik, M. Baker, LTE, The UMTS Long Term Evolution: From Theory to Practice, Wiley Publishing, 2009.

[24] A. Marini, Performance evaluation of different scheduling algorithms in LTE systems, in: 2016 39th International Convention on Information and Communication Technology, Electronics and Microelectronics (MIPRO), 2016, pp. 595-600. doi:10.1109/MIPRO.2016.7522211. 


\section{Vitae}

A. Burbano-Abril received the BSc degree in Electronic Engineering from the University of Azuay (UDA) in Ecuador and the MSc (Hons) degree in Electrical Engineering (Communications) from the National Autonomous University of Mexico (UNAM) in 2013 and 2016, respectively. His current research focuses on data science applications for 5G networks. He is currently a Ph.D. student at UNAM.

R. M. Edwards received his degree in Electronic Engineering (Communications) from the University of Sheffield, and then studied for a Ph.D. with the Department's internationally respected Communications and Radar Group. He is now director of the 5G Research Centre at Loughborough University UK and is the lead in the universities Mobile Communications expertise.

V. Rangel-Licea received the BSc (Hons) degree in computer engineering from the National Autonomous University of Mexico (UNAM) in 1996. He received the MSc and Ph.D. degrees from the University of Sheffield, United Kingdom, in 1998 and 2002, respectively. His research focuses on QoS, traffic modeling, and scheduling for mobile broadband wireless access networks. He has been with the School of Engineering (UNAM) since 2002 as a research professor.

R. Aquino-Santos studied Electrical Engineering at the University of Colima from 1983 to 1988 . He completed postgraduate studies in Applied Physics in Electronic and Telecommunications at the Center for Scientific Research and Higher Education of Ensenada (CICESE) from 1988 to 1990. From 2000 to 2005, Raúl Aquino carried on his doctoral studies at the University of Sheffield, England. Currently, his areas of interest are the design, development, simulation and implementation of wireless telecommunication networks: IoT, and Industry 4.0.

M. Lopez-Guerrero received his Ph.D. in electrical engineering from the University of Ottawa (Canada) in 2004. Currently, he is with Metropolitan Autonomous University (Mexico), Iztapalapa campus. His research interests are related to several aspects of communication networks such as data traffic modeling, medium access control and performance evaluation.

R.S. Kalawsky is Professor of Systems Engineering and Human Computer Integration and Director of the Advanced VR Research Centre. He was the Director of the Research School of Systems Engineering and Technical Head of the Systems Engineering Innovation Centre. He headed up the Systems Division within the Department of Electronic and Electrical Engineering, before becoming Associate Dean.

M. Behjati received the Ph.D. in Electrical Engineering and Electronics, Wireless Communications, from Brunel University London in 2015. He is the winner of the Walduck Prize for Research Impact in year 2015 in the UK. Dr. Behjati has also worked as a Postdoctoral Researcher at the 5G Research Centre, Loughborough University, UK. 